\title{
Management of Epilepsy among Children in the Gaza Strip: A Multicenter Clinical Audit
}

\author{
Tayseer Afifi Khamis Elessi Mosab Samaan \\ Ahmed Abu-Lamzi \\ Faculty of Medicine, The Islamic University - Gaza, Gaza, Palestine
}

\section{Keywords \\ Epilepsy · Child · Clinical audit · Gaza · Neurology}

\begin{abstract}
Introduction: Seizures are scary phenomena but are quite common especially among children: about $8 \%$ will have had at least one seizure by 15 years of age. Most acute seizures in children are brief, terminating spontaneously and do not need any treatment. Seizures that persist beyond 5 min may not stop spontaneously and necessitate comprehensive clinical evaluation and medical care. Seizure control then becomes a matter of urgency for the patient and his family, as the longer the duration of the seizure, the greater the bad effects on body systems especially the brain and the more difficult control is likely to become. Methods: A retrospective, multicenter clinical audit was undertaken simultaneously at the three largest centers for epilepsy management in Gaza. A total of 190 medical records were included in our audit from January 1, 2017, to April 30, 2018. Aims and Objectives: Our audit aims to evaluate the care provided for patients with epilepsy from diagnosis to treatment and follow-up and to compare it against the latest National Institute for Health and Care Excellence (2017) guidelines and AACC
\end{abstract}

(American Association for Clinical Chemistry) (January 7, 2018). Results: Among 190 reviewed medical records, 61\% patients were males and the median age was 9 ranging from 6 to 13 years. $31.5 \%(n=60)$ of cases suffered from various comorbidities. The overall adherence of health care professionals to audit standards was moderate. There was severe dearth of information in the medical records. Most records ( $n=147,77.4 \%$ ) did not include the seizure type, frequency, and duration. Drug therapy was inconsistent with seizure type in 16 cases (8.4\%). Investigations were underutilized generally. There were marked differences in the practice among health care centers. Conclusion: The vast majority of files ( $n=138,72.6 \%$ ) suffered from poor documentation of seizure type, frequency, and duration which needs to be improved. Drug prescription was inconsistent and highly variable and dependent on the treating doctor's memory and diligence. Moreover, electroencephalogram, electrocardiogram, and drug level testing were highly suboptimal. Health professionals should modify and adopt the AACC recommendations to improve diagnostic and therapeutic service and reduce potential complications and sufferings of epileptic patients.

(C) 2019 The Author(s) Published by S. Karger AG, Basel

\begin{tabular}{ll}
\hline KARGER & $\begin{array}{l}\text { () } 2019 \text { The Author(s) } \\
\text { Published by S. Karger AG, Basel Oparger }\end{array}$ \\
E-Mail karger@karger.com & This article is licensed under the Creative Commons Attribution- \\
www.karger.com/dmj & $\begin{array}{l}\text { NonCommercial-NoDerivatives 4.0 International License (CC BY- } \\
\text { NC-ND) (http://www.karger.com/Services/OpenAccessLicense). } \\
\text { Usage and distribution for commercial purposes as well as any dis- } \\
\text { tribution of modified material requires written permission. }\end{array}$
\end{tabular}

Tayseer Jamal Afifi

Faculty of Medicine, The Islamic University - Gaza Abu Alsaeed

Rafah, Gaza Strip 79794 (Palestine)

E-Mail afifi.tayseer@gmail.com 


\section{Introduction}

Epilepsy is a common and serious neurological disorder in childhood. In reality it is not a single disease but comprises a range of electroclinical phenotypes that result from many underlying disorders. Children with epilepsy differ from adults not only in the clinical manifestations of their seizures, but also in the presence of unique electroencephalogram (EEG) patterns, etiologies, and response to antiseizure drugs [1]. They are more prone to seizures, but seizures are more about to disappear as the child grows. The management of the care of children with epilepsy has important implications for all aspects of their daily lives, especially daily activities such as bathing, vocational and leisure pursuits. There are areas of continuing concern with regard to therapy and compliance. Previous studies [2,3] have shown that there is great potential for improving the management of these children, with particular reference to accurate diagnosis, appropriate therapeutic intervention, and well-documented, careful, ongoing support.

There has been long-standing concern regarding misdiagnosis and quality of management. Thus, this paper reflects on the current practice and further to improve the care delivered in Gaza Strip health care centers.

Additionally, through this work we wanted (I) to document the seizure frequency and appropriateness of daily therapy to aid patient compliance, (II) to ascertain the laboratory tests requested and their frequencies, and (III) to propose strategies to improve these aspects of epileptic care.

\section{Materials and Methods}

This study was carried out in the three largest centers for epilepsy management in the Gaza Strip, namely: European Gaza Hospital, Nasser Medical Complex, and Rantisi Pediatric Hospital. The study involved the medical records of 190 children seen at a pediatric neurology department during the period from January 1 , 2017, to April 30, 2018.

We chose a clinically representative rather than a randomized controlled sample as the present study aimed to evaluate the performance of the health care professionals.

Medical records with insufficient information on the child, or when laboratory results or prescribed drug lists were missed were excluded from the study since the assessment depends largely on these items.

Principles for the management of these patients were devised from two sources with advice from two neurologists. Validated international standards used for this study were chosen from these principles. Through an audit process, the assessed aspects of care were: (1) seizure documentation, duration and frequency, (2) requested investigations which included imaging studies, blood tests and chemistry, and (3) treatment.

Management of Epilepsy among Children
International Standards

The latest National Institute for Health and Care Excellence (NICE) (2018) guidelines and American Association for Clinical Chemistry (AACC) (2017) were followed in this audit $[4,5]$.

Laboratory and nonlaboratory tests are used to diagnose and monitor epilepsy, to determine what kinds of seizures a child is having, to identify underlying conditions such as toxins, infections or fever in children that may be causing seizures, and to distinguish epilepsy from conditions such as fainting that may cause some of the same symptoms.

A medical history, input from the patient, and input from family members who have witnessed the seizures are important parts of the diagnostic process. Someone with epilepsy may remember a strange smell, an aura, and/or sensations that precede a seizure but may not remember what has happened during the seizure itself. Depending upon the signs and frequency of a child's seizures, it may take some time to determine the proper diagnosis. Table 1 demonstrates the best available recommendations for practice.

\section{Data Collection}

Five medical students were trained for 1 week on study proto$\mathrm{col}$, tools and data collection techniques of the study. Data were collected using a self-designed questionnaire based on the NICE and AACC guidelines. The survey tool encompassed questions related to demography and socioeconomy, presence of comorbidities, diagnostic information and treatment prescribed for the case. The data collection sheet was revised after conducting a pilot survey among 15 medical records. The data were collected retrospectively choosing the first completed files, which contained the adequate information we needed. The study focused on all known children with epilepsy in the three centers.

\section{Definitions of Variables}

Children were selected for this study as younger than 15 years old. Medical records were chosen as they had the ICD number G40 which indicates the diagnosis of epilepsy.

\section{Statistical Analysis}

The collected data were initially entered to Microsoft Excel. Then, data were analyzed by SPSS version 23 . Frequency distributions and percentages were computed for all the variables.

\section{Ethical Consideration}

Ethical clearance was obtained from the Directorate General of Human Resources Development at the Ministry of Health, State of Palestine. Written and verbal consents were gained form health care centers' heads to enable us to review medical records. Study objectives, data collection procedures, benefits and risks of the study, confidentiality, and anticipated use of the results were explained to the research committee in the ministry in detail before executing our work.

\section{Results}

\section{Baseline Characteristics}

Our study included the revision of medical records of 190 children diagnosed with epilepsy in three hospi- 
Table 1. Key recommendations for practice [6-16]

\begin{tabular}{lll}
\hline Specifics addressed & Basis of recommendations & Key recommendations \\
\hline $\begin{array}{l}\text { Management of pediat- } \\
\text { ric status epilepticus }\end{array}$ & $\begin{array}{l}\text { Expert opinion; not system- } \\
\text { atic review }\end{array}$ & $\begin{array}{l}\text { First-line treatment benzodiazepines (either diazepam or lorazepam) } \\
\text { Second-line treatment phenytoin or fosphenytoin OR phenobarbital OR valproate (intrave- } \\
\text { nous) } \\
\text { with } \\
\text { intensive-care unit care including ventilation } \pm \text { EEG monitoring } \pm \text { invasive hemodynamic } \\
\text { monitoring }\end{array}$
\end{tabular}

\begin{tabular}{ll}
\hline Seizures including par- & Italian League against Epilep- \\
tial seizures (simple & sy systematic evidence-based \\
complex and secondari- & review
\end{tabular}
complex and secondari- review ly generalized), and generalized tonic and/or clonic seizures

History and examination warranted though only observational studies support this

Investigations recommended:

Complete blood count

Glucose

Urea

Electrolytes

Calcium

Creatinine

Alanine and aspartate aminotransferases

Creatine kinase/prolactin

Urine analysis

Toxicological tests

With evidence in case-control and cohort studies

For infants, assessing for hyponatremia has high-level evidence from meta-analysis studies Imaging with CT or MRI and EEG recommended based upon level 1 evidence with prospective cohort studies, clinical trials or meta-analysis

Diagnostic assessment Evidence-based review by of child with status American Academy of Neuepilepticus rology and Child Neurology Society

Treatment of status $\quad$ Finnish Neurologic Society
Insufficient data to support blood cultures or lumbar puncture unless there is clinical suspicion of systemic or CNS infection and insufficient data to routinely recommend neuroimaging Checking AED levels in child on an AED was considered "probably effective"

The following were determined to be "possibly effective": toxicology studies, metabolic studies for inborn errors of metabolism, EEG for nonconvulsive status epilepticus

First line: buccal midazolam, rectal diazepam, or intravenous diazepam or lorazepam Second line: phenobarbital

Third line: suppressive anesthesia with continuous EEG

Generalized convulsive Canadian Paediatric Society status epilepticus in children providing care guidelines while acknowledging there are insufficient data to guide care. Pragmatic expert advice

Check glucose then $\mathrm{ABC}$ including positioning, suctioning, oxygen, and low threshold for ventilation

First line with intravenous treatment is lorazepam, diazepam or midazolam; if no intravenous line, then buccal lorazepam, buccal or intranasal lorazepam, or rectal diazepam

Second line intravenous fosphenytoin, phenytoin or phenobarbital; if IO then fosphenytoin, phenytoin, or paraldehyde

Refractory status with intravenous midazolam or pentobarbital

Generalized convulsive Expert opinion noting there status epilepticus are no nationally recognized specific protocols in North America

Initially check glucose and ABCs; intubation and ventilation; ceftriaxone or vancomycin pending head CT and LP; electrolytes and chemistries needed. EEG if nonconvulsive status epilepticus of concern

Treatment with intravenous diazepam or lorazepam immediately; then phenytoin or fosphenytoin; if further treatment is needed, phenobarbital or valproate

For refractory status, continuous infusion with midazolam or propofol

Treatment of convulsive Italian League against Epilepstatus epilepticus sy systematic evidence-based review

First line preferred via intravenous lorazepam or diazepam; if no intravenous access, then intramuscular or buccal midazolam or buccal lorazepam based upon RCT or meta-analysis of RCT Second line phenytoin or phenobarbital or valproate based upon at least one RCT

For refractory status epilepticus, they recommend thiopental or propofol or midazolam based upon expert opinion

Multi-Disciplinary Group for Diagnostics to include total and ionized calcium in those $<2$ years old, sodium in those $<6$ the Management of Status Epilepticus in Children in India months old, and complete blood count and LP if febrile; second-line diagnostics to include EEG and imaging

$\mathrm{ABC}$ with oxygen and continuous positive airway pressure or invasive ventilation if needed First-line treatment intravenous lorazepam, diazepam or midazolam. If no intravenous treatment, then intramuscular/intranasal/buccal midazolam or rectal diazepam

Second-line treatment phenytoin, fosphenytoin, phenobarbital or valproate. If this fails, pyridoxine in $<2$ years old; otherwise, levetiracetam

Refractory status midazolam or thiopental 
Table 1 (continued)

\begin{tabular}{|c|c|c|}
\hline Specifics addressed & Basis of recommendations & Key recommendations \\
\hline $\begin{array}{l}\text { Monitoring oxygen } \\
\text { saturations, providing } \\
\text { supplemental oxygen, } \\
\text { evaluation and treat- } \\
\text { ment of acute seizures }\end{array}$ & $\begin{array}{l}\text { World Health Organization's } \\
\text { Paediatric Emergency Triage, } \\
\text { Assessment and Treatment } \\
\text { Group }\end{array}$ & $\begin{array}{l}\text { Re: monitoring oxygenation - strong recommendation with low quality of evidence for moni- } \\
\text { toring given low risk } \\
\text { Re: oxygen use - conditional recommendation with low quality of evidence for use of oxygen } \\
\text { with judicious use and lower threshold for discontinuation where supplemental oxygen is a rare } \\
\text { resource } \\
\text { Re: diagnostic assessments with acute seizures - strong recommendation with low quality of } \\
\text { evidence for checking glucose, sodium if there is dehydration or diarrhea; also for LP if febrile } \\
\text { particularly for those }<18 \text { months old, those with complex seizures, prior antibiotic use or not } \\
\text { vaccinated for Haemophilus influenzae and/or streptococcal meningitis; LP deferral if unstable; } \\
\text { image if new focal findings or comatose } \\
\text { First-line treatment with intravenous access has strong recommendation based on low-quality } \\
\text { evidence for treatment with benzodiazepine but condition recommendation on choice of agent } \\
\text { with these being midazolam, lorazepam or diazepam, where intravenous access; where there is } \\
\text { no established intravenous access, there are strong recommendations based upon low evidence } \\
\text { for rectal diazepam, oral or intranasal midazolam, rectal or intranasal lorazepam, or intramus- } \\
\text { cular lorazepam or midazolam } \\
\text { Second-line treatment is a conditional recommendation based upon low-quality evidence for } \\
\text { phenytoin or phenobarbital or valproate }\end{array}$ \\
\hline $\begin{array}{l}\text { Compared anticonvul- } \\
\text { sant treatments for } \\
\text { convulsive status epilep- } \\
\text { ticus }\end{array}$ & $\begin{array}{l}\text { Evidence-based guidelines } \\
\text { from American Epilepsy } \\
\text { Society }\end{array}$ & $\begin{array}{l}\text { Of } 38 \text { studies and RCTs, only } 4 \text { provided level } 1 \text { evidence ("established as effective") based upon } \\
\text { blinded RCT; } 2 \text { studies provided evidence "established as probably effective" from one or more } \\
\text { unblinded or otherwise limited RCT; the remainder of the RCTs or observational studies pro- } \\
\text { vided evidence of "possible" effectiveness } \\
\text { Established as effective included intravenous lorazepam or intravenous diazepam for acute } \\
\text { seizure management; there was insufficient evidence for superiority of lorazepam vs. diazepam } \\
\text { Established as possibly effective was rectal diazepam, intramuscular midazolam, intravenous } \\
\text { midazolam, buccal midazolam } \\
\text { For second-line treatment, fosphenytoin is better tolerated than phenytoin }\end{array}$ \\
\hline $\begin{array}{l}\text { Management of status } \\
\text { epilepticus }\end{array}$ & $\begin{array}{l}\text { Expert panel developed con- } \\
\text { sensus; no evidence ratings } \\
\text { given }\end{array}$ & $\begin{array}{l}\text { ABC with high concentration oxygen; check glucose and establish intravenous access } \\
\text { First line with intravenous access is lorazepam; if lorazepam is not available then diazepam; if } \\
\text { no intravenous access, then buccal midazolam } \\
\text { Second line phenobarbital or phenytoin } \\
\text { Refractory status with midazolam or thiopental }\end{array}$ \\
\hline
\end{tabular}

AED, antiepileptic drugs; ABC, airway, breathing and circulation; LP, lumbar puncture; RCT, randomized controlled trial; IO, intraosseous.

tals in the Gaza Strip. Based on the analysis of the whole cohort, $60.5 \%(n=115)$ were males, and the median age was 9 ranging from 6 to 13 years old; however, the median age at which the first seizure appeared was 5 years. The children involved in the study suffered from many comorbidities, $54 \%(n=103)$ of cases suffered from pneumonia, asthma and other etiological conditions of epilepsy. Regarding seizure type, $12.5 \%$ of cases $(n=24)$ had tonic-clonic seizure, $3.5 \%(n=7)$ had febrile seizure, $2.5 \%(n=5)$ had generalized tonic seizure, and $3.5 \%(n=7)$ had other types. On the other hand, $77.5 \%$ of cases $(n=147)$ had no documentation regarding seizure type. Furthermore, the seizure frequency was not documented at all. Table 2 summarizes the results for each center.

\section{Investigations}

EEG, the primary diagnostic tool for epilepsy, was done in 49 out of 190 patients (25.7\%). NICE guidelines recommend doing EEG for all epileptic patients to determine the type of seizure. An electrocardiogram (ECG) was investigated in $7 \%$ of patients $(n=13)$ only; the NICE guideline recommends that a standard 12-lead ECG should be done in all patients presenting with suspected seizure. Drug levels have been monitored in only $19.5 \%$ of cases $(n=37)$. According to the AACC guideline, clotting tests must be studied in patients on valproic acid. In spite of that, only 14 (7.4\%) patients had been tested. Figure 1 shows how frequently investigations were requested in the actual practice. 
Table 2. Characteristics of study subjects and distribution of comorbidities

\begin{tabular}{|c|c|c|c|c|}
\hline Study variables & $\begin{array}{l}\text { EGH, } \\
\%(n)\end{array}$ & $\begin{array}{l}\text { NMC, } \\
\%(n)\end{array}$ & $\begin{array}{l}\mathrm{RPH} \\
\%(n)\end{array}$ & $\begin{array}{l}\text { Total, } \\
\%(n)\end{array}$ \\
\hline Total number of cases & $23.5(45)$ & $34(65)$ & $42.5(80)$ & $100.0(190)$ \\
\hline \multicolumn{5}{|l|}{ Seizure type } \\
\hline Generalized tonic & $4.5(2)$ & & $3.75(3)$ & $2.5(5)$ \\
\hline Tonic-clonic & $18(8)$ & $23(15)$ & $1.25(1)$ & $12.5(24)$ \\
\hline Myoclonic & & $1.5(1)$ & $1.25(1)$ & $1.05(2)$ \\
\hline Febrile seizure & $2(1)$ & $9(6)$ & & $3.5(7)$ \\
\hline Focal seizure & $4.5(2)$ & & & $1.05(2)$ \\
\hline Absence seizure & $2(1)$ & & & $0.5(1)$ \\
\hline Status epilepticus & & $3(2)$ & & $1.05(2)$ \\
\hline Not documented & $69(31)$ & $63(41)$ & $93.75(75)$ & $77.5(147)$ \\
\hline \multicolumn{5}{|c|}{ Comorbidities and etiological conditions } \\
\hline Pneumonia & $29(13)$ & & $18.75(15)$ & $15.0(28)$ \\
\hline Sanjad-Sakadi syndrome & $4.5(2)$ & & & $1.05(2)$ \\
\hline Cerebral palsy & $82(37)$ & $1.5(1)$ & $1.25(1)$ & $20.5(39)$ \\
\hline Celiac disease & $2(1)$ & & & $0.5(1)$ \\
\hline Asthma & $4.5(2)$ & & $3.75(3)$ & $2.5(5)$ \\
\hline Angelman syndrome & $2(1)$ & & & $0.5(1)$ \\
\hline Brain atrophy & $11 \%(5)$ & $6 . \%(4)$ & & $4.5(9)$ \\
\hline Hepatitis A & $2(1)$ & & & $0.5(1)$ \\
\hline Familial Mediterranean fever & $2(1)$ & & & $0.5(1)$ \\
\hline Cystic fibrosis & $2(1)$ & & & $0.5(1)$ \\
\hline Acute bronchiolitis & & $5(3)$ & $1.2(1)$ & $2.0(4)$ \\
\hline Down syndrome & & & $2.5(2)$ & $1.05(2)$ \\
\hline Immunodeficiency & & & $1.25(1)$ & $1.05(2)$ \\
\hline Neurodegenerative disease & & & $1.25(1)$ & $0.5(1)$ \\
\hline
\end{tabular}

One child may have more than one comorbidity. Empty cells mean none or zero. EGH, European Gaza Hospital; NMC, Nasser Medical Complex; RPH, Rantisi Pediatric Hospital. For percentages presented in the field under each hospital, the number is divided by the total of the hospital. For percentages in the total column, the numbers are divided by 190 .

\section{Drug Therapy}

Interestingly, $25 \%$ of cases $(n=48)$ were under no therapy. However, $42.5 \%(n=81)$ were under polytherapy and $32 \%(n=61)$ were under monotherapy (Fig. 2). Valproic acid was the most prescribed drug. It was prescribed in $36 \%$ of cases $(n=67)$, followed by phenobarbital, phenytoin, and diazepam; they were collectively prescribed in $62 \%$ of cases $(n=118)$. By contrast, the drug was not matched with the type of seizure in $8.5 \%$ of cases as compared to the NICE guidelines. Drug levels are of important concern in epilepsy patients. However, only $19.5 \%$ of cases $(n=37)$ had their drug level being monitored during therapy. In summary, Figure 3 demonstrates the number of drug prescriptions in the practice. It is well observed in Figure 2 that health care providers depended largely on a combination between antiepileptic drugs. Most of these combinations were between phenobarbital and valproic acid in $31.5 \%$ of cases $(n=60)$ and between clonazepam and diazepam in $11 \%$ of patients. As previously mentioned, as most children suffered from various comorbidities, they were admitted several times to the hospital. As a result, they were also prescribed drugs for their conditions. Antibiotics were on the top of the list followed by ranitidine and nonsteroidal antiinflammatory drugs. 


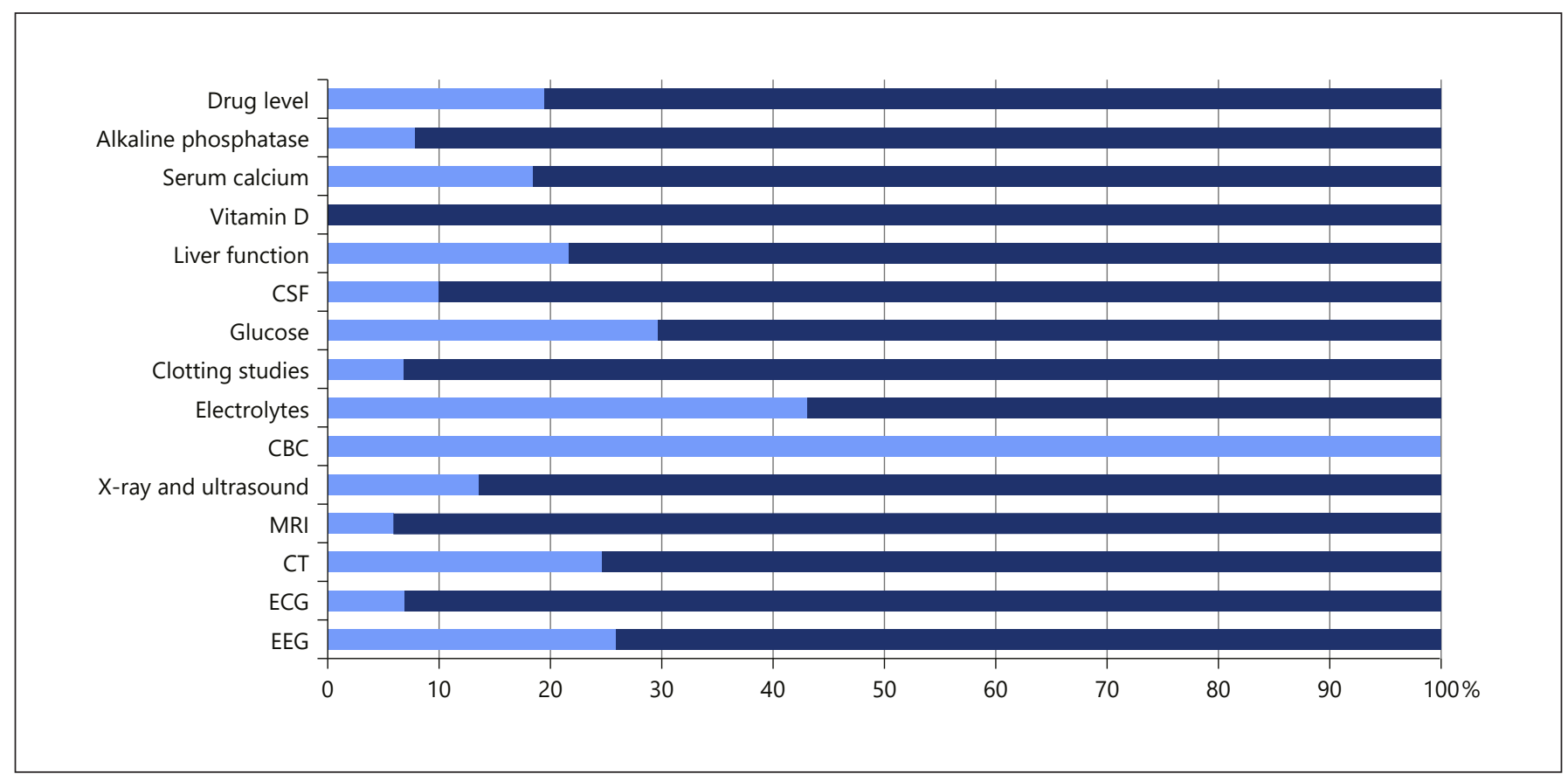

Fig. 1. Percentages of requested tests.

\section{Adherence to Guidelines}

It is clearly observed that the practice of health care providers in Gaza Strip centers is not highly committed to the guidelines. The practice varied among the three centers in almost all categories of the standards. However, there were some mutual deficits in the practice in all centers. For example, all files suffered from poor documentation of basic information such as seizure type, seizure frequency, and duration. On the other hand, the centers differed in drug prescriptions and combinations. Overall, their adherence to the adopted standards was found only in $29 \%$ of cases $(n=55)$.

\section{Discussion}

To the best of our knowledge, this is the first audit in Palestine concerned with epilepsy in children. However, there is no reliable statistics regarding the incidence of epilepsy among children. Here, our work had some criteria for selecting the medical records, and we did not include every single record of epileptic patients. The information we sought to evaluate in the medical records was half-heartedly demonstrated. This was the barrier as we did not include all patient files.

Management of Epilepsy among Children
It is well recognized that documentation of seizure type, frequency, and duration is poor. The absence of documentation suggests that epilepsy was not the focus of the consultation. The reasons for this are likely to have been that the patients were consulting for reasons other than epilepsy, that their condition was stable, or that there was not enough time available during the consultation. It may be that neither patients nor doctors wish to draw attention to this stigmatized condition.

As mentioned above, most patients suffered from other comorbidities. Moreover, investigations are underutilized, and appropriate laboratory tests as well as imaging studies were not requested occasionally. In addition, the unavailability of some lab tests and lab technicians had a great impact on utilization of these facilities.

Drug prescription was inconsistent and highly variable and dependent on the treating doctor's memory and diligence. There is large dependence on some medications due to their availability. On the other hand, some medications should be prescribed, but the health system lack them, or the family of the child cannot afford them.

As the diagnosis of the epilepsy type is not accurate, the treatment plan will not be convenient. And the treatment period is prolonged without noticeable development in the case condition. However, health care provid- 
Fig. 2. Distribution of drug therapy.
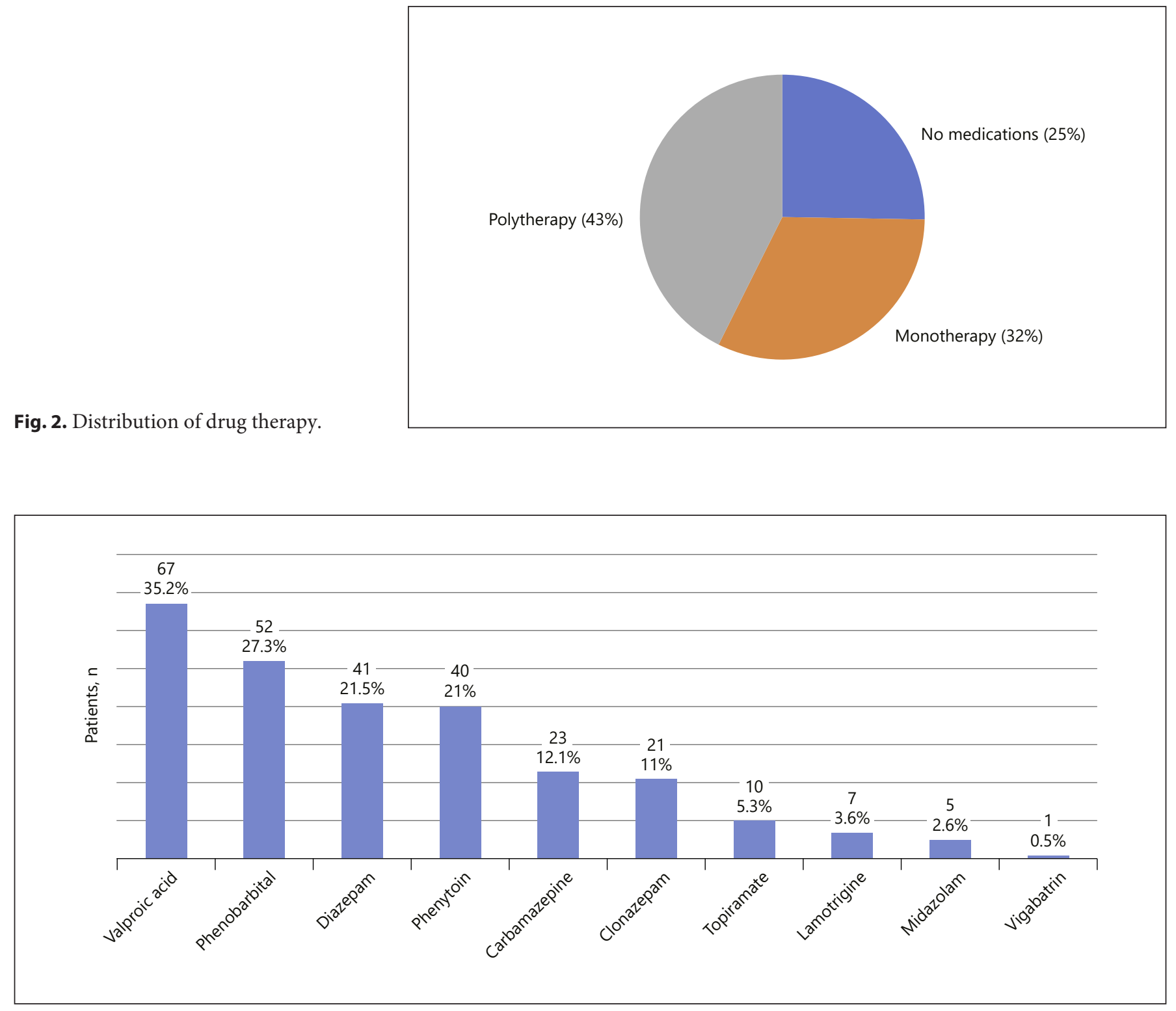

Fig. 3. Number of antiepileptic drug prescriptions. One patient may be prescribed more than one drug.

ers were aware enough in changing the medication and about drug combination. Thus, we did not record any complicated condition.

It is clearly obvious that the practice in Gaza Strip centers is suboptimal. So, as a result of this audit, further developments are planned. Successive audits and close monitoring of the practice will dramatically lead to better documentation and proper use of investigations. This in turn will fulfill the gap of incorrect diagnosis. This eventually will aid in suitable drug prescriptions.
During our work, we observed marked variation in the practice among the centers and this is due to the lack of agreement between health care providers on the best standards to follow. However, the measures in the previous paragraph could remedy this gap.

Studies on children with epilepsy in regional countries have provided some useful statistics. A study in Saudi Arabia [17] found the prevalence rate of epilepsy in children is $6.54 / 1,000$ in 2001 by door-to-door survey. The study also reported that perinatal and inherited factors 
are the predominant causes of symptomatic epilepsy. Another study conducted in Sudan as a low-resource country as the Gaza strip concluded that epilepsy continues to be a major public health problem with a considerable gap in its treatment. The study also added there is a need for rational use of investigation when required. Furthermore, the study addressed the importance of public knowledge and attitude improvement [18]. The previous recommendations are similar to those of our study.

This study shows that annual review and seizure frequency documentation are required in order to improve the medical records, but alterations in drug therapy are more difficult. Barriers to change exist, but it is proposed that they can be overcome by successive audits based on a primary care team approach with close collaboration with a neurologist. Health professionals should modify and adopt the AACC recommendations to improve diagnostic and therapeutic service and reduce potential complications and sufferings of epileptic patients.

\section{Conclusion}

Epilepsy management in Gaza Strip centers is clearly suboptimal compared to internationally validated practices particularly in the fields of completing documentation and the proper use of investigations and medications. A specialized epilepsy clinic should be the focal point for acute and follow-up care. Traditionally, the main player in this care is the epilepsy experts; however, overall care improvement requires better collaboration between neurologists and other general practitioners who can make a considerable contribution to continued patients care. The authors suggest that a local protocol of management of epilepsy should be drafted based on vali- dated guidelines. It is also suggested that local epilepsy registers should be created to enable long-term surveillance and research.

Finally, a refreshing course on the management of epilepsy has to be conducted for concerned staff followed by a successive audit to measure improvements.

\section{Acknowledgment}

We thank our faculty for assistance with getting permission to commence the study, and archive staff for their help in extracting files for reviewing.

\section{Statement of Ethics}

Ethical clearance was obtained from the Directorate General of Human Resources Development at the Ministry of Health, State of Palestine. Written consent was gained form health care centers' heads to enable us to review medical records. Study objectives, data collection procedures, benefits and risks of the study, confidentiality, and anticipated use of the results were explained to the research committee in the ministry in detail before executing our work.

\section{Disclosure Statement}

The authors declare that they have no competing interests.

\section{Author Contributions}

T.A. and K.E. designed the study questionnaire. T.A., A.A., H.A.E., A.A.L., and M.S. collected the data. Data analysis was done by M.S. and T.A. T.A. wrote the first draft of the manuscript. K.E. revised it for grammatical mistakes and reviewed the statistics and figures. K.E. supervised the overall process and reviewed the final draft. All authors approved the final version of the manuscript.

\section{References}

1 Ngugi AK, Bottomley C, Kleinschmidt I, Sander JW, Newton CR. Estimation of the burden of active and life-time epilepsy: a meta-analytic approach. Epilepsia. 2010 May; 51(5):883-90.

2 Brown S, Betts T, Chadwick D, Hall B, Shorvon S, Wallace S. An epilepsy needs document. Seizure. 1993 Jun;2(2):91-103.

3 Cooper GL, Huitson A. An audit of the management of patients with epilepsy in thirty general practices. J R Coll Gen Pract. 1986 May;36(286):204-8.

Management of Epilepsy among Children
4 Epilepsy: diagnosis and management. April 2018 [cited Jan 2019]. Available from: https:// www.nice.org.uk/guidance/cg137.

5 Epilepsy. October 26, 2017 [cited Jan 2019]. Available from: https://labtestsonline.org/ conditions/epilepsy.

6 Glauser T, Shinnar S, Gloss D, Alldredge B, Arya R, Bainbridge J, et al. Evidence-based guideline: treatment of convulsive status epilepticus in children and adults: report of the Guideline Committee of the American Epilepsy Society. Epilepsy Curr. 2016 Jan-Feb; 16(1):48-61.
7 Kälviäinen R, Eriksson K, Parviainen I. Refractory generalised convulsive status epilepticus : a guide to treatment. CNS Drugs. 2005; 19(9):759-68.

8 Beghi E, De Maria G, Gobbi G, Veneselli E. Diagnosis and treatment of the first epileptic seizure: guidelines of the Italian League against Epilepsy. Epilepsia. 2006;47(s5 Suppl 5):2-8. 
9 Riviello JJ Jr, Ashwal S, Hirtz D, Glauser T, Ballaban-Gil K, Kelley K, et al.; American Academy of Neurology Subcommittee; Practice Committee of the Child Neurology Society. Practice parameter: diagnostic assessment of the child with status epilepticus (an evidence-based review): report of the Quality Standards Subcommittee of the American Academy of Neurology and the Practice Committee of the Child Neurology Society. Neurology. 2006 Nov;67(9):1542-50.

10 Kälviäinen R, Eriksson K, Häppölä O, Keränen T, Kurola J, Kuusela AL, et al.; Suomalaisen Lääkäriseuran Duodecimin; Suomen Lastenneurologinen Yhdistys RY:n; Suomen Neurologinen Yhdistys RY:n Asettama Työryhmä. [Update on current care guidelines. The treatment of status epilepticus]. Duodecim. 2009;125(22):2469-71. In Finnish.
11 Friedman J. Emergency management of the paediatric patient with generalized convulsive status epilepticus. Paediatr Child Health. 2011 Feb;16(2):91-104.

12 Shearer P, Riviello J. Generalized convulsive status epilepticus in adults and children: treatment guidelines and protocols. Emerg Med Clin North Am. 2011 Feb;29(1):51-64.

13 Capovilla G, Beccaria F, Beghi E, Minicucci F, Sartori S, Vecchi M. Treatment of convulsive status epilepticus in childhood: recommendations of the Italian League Against Epilepsy. Epilepsia. 2013 Oct;54 Suppl 7:23-34.

14 Mishra D, Sharma S, Sankhyan N, Konanki R, Kamate M, Kanhere S, et al.; Multi-disciplinary Group on Management of Status Epilepticus in Children in India. Consensus guidelines on management of childhood convulsive status epilepticus. Indian Pediatr. 2014 Dec; 51(12):975-90.
15 Updates on paediatric emergency triage, assessment and treatment: care of critically ill children. Geneva: World Health Organization; 2016.

16 Bashiri FA, Hamad MH, Amer YS, Abouelkheir MM, Mohamed S, Kentab AY, et al. Management of convulsive status epilepticus in children: an adapted clinical practice guideline for pediatricians in Saudi Arabia. Neurosciences (Riyadh). 2017 Apr;22(2): 146-55.

17 Al Rajeh S, Awada A, Bademosi O, Ogunniyi A. The prevalence of epilepsy and other seizure disorders in an Arab population: a community-based study. Seizure. 2001 Sep;10(6): 410-4.

18 Eltahir Babiker MO. Managing childhood epilepsy in a resource-limited setting: A pragmatic approach. Sudan J Paediatr. 2015;15(1): 9-20. 